\title{
The Effect of Pressure on Thermal Stability and In Vitro Fibril Formation of Collagen
}

\author{
Kunihiko GekKo and Shozo KogA \\ Department of Food Science and Technology, Faculty of Agriculture, \\ Nagoya University, Nagoya 464, Japan
}

Received October 22, 1982

\begin{abstract}
The effects of pressure on thermal denaturation and in vitro fibril formation of acid soluble collagen from calf skin were studied with various solvent conditions under high pressure of up to 4,000 atm. For all the solvent systems, the thermal denaturation was depressed by pressure, e.g., the apparent denaturation temperature at $\mathrm{pH} 4.0$ increased from $37.5^{\circ} \mathrm{C}$ at 1 atm to $44.5^{\circ} \mathrm{C}$ at 1,400 atm. The pressure-enhanced thermal stability of collagen indicates that the volume change due to the helix-to-coil transition of this protein is positive in water. The fibril formation in both the nucleation and growth phases was extensively retarded by pressure in a relatively low region of up to $500 \mathrm{~atm}$, indicating the positive activation volume of this fibril formation process. These results seemed to support a hypothesis that the protein-water interaction plays a dominant role in the structural stability of the triple helix and fibrils of collagen.
\end{abstract}

Water has been expected to be essential for the maintenance of triple helix and fibril structures of collagen. The existence of localized water molecules around a collagen molecule has often been detected by some physicochemical techniques. ${ }^{1 \sim 3)}$ Recent X-ray analyses of protein crystals have shown that hydrogen-bonded bridges involving more than one water molecule may be formed to link the $\mathrm{C}=\mathrm{O}$ groups of the glycine and $\mathrm{Y}$-residues in the collagen unit $(\mathrm{Gly}-\mathrm{X}-\mathrm{Y})_{n}{ }^{4,5)}$ However, there is not enough evidence demonstrating to what extent protein-water interactions really contribute to the stability of collagen structures, while such contribution has been suggested from solvent perturbation studies $^{6 \sim 9)}$ and related discussions. ${ }^{10}$ Privalov and Tiktopulo $^{11)}$ pointed out that their calorimetric data on thermal denaturation of tropocollagen could not be explained with the assumption that the triple helix is stabilized only by the intramolecular hydrogen bonds or by the restriction of backbone rotation with the pyrrolidine residues. They attributed the dominant stabilizing force of the collagen triple helix to the water bridges in the vicinity of the imino acid groups and to several layers of water adjacent to the localized water molecules, while Cooper proposed that the water structure plays an important role in the fibril formation of collagen in addition to various solvent factors such as $\mathrm{pH}$ and ionic strength. ${ }^{12)}$ Since this process is an endothermic reaction, it must be driven by a relatively large increase in entropy, presumably caused by reorientation of water molecules in the vicinity of the protein.

Despite the extensive data on temperature effects, the influence of pressure on the stability of collagen structures has remained unknown probably due to experimental difficulties, while the trans-cis transition of poly-Lproline, a model polymer of collagen, was examind and found to be affected by pressure. ${ }^{13)}$ In most cases, the volume change due to the helix-to-coil transition of collagen has not been taken into consideration or has been assumed to be negligibly small, ${ }^{14)}$ although there is a priori no reason to set the pressure work term $P \Delta V$ equal to zero unless the experiments are done at constant volume. This assumption seems to be unreasonable since the amount of hydration of collagen is different in its native and denatured states. ${ }^{15)}$ Some direct 
measurements have been carried on the volume changes due to the helix-coil transition and fibril formation of collagen, but the results seem to be inconsistent with each other. ${ }^{16 \sim 18)}$ In this connection, high pressure experiments should be a diagnostic test for such volume effects or the participation of water in stabilizing collagen structures. Even if done in vitro, they also would give basic information on the effect of the environmental pressure of animals on the structural stability of their collagens.

From these viewpoints, we have examined the effects of hydrostatic pressure on thermal denaturation and fibril formation of acid soluble collagen in aqueous media. This paper is devoted to the experimental results and to discussion of the stabilization mechanism of collagen structures in terms of protein-solvent interactions.

\section{MATERIALS AND METHODS}

Materials. Two types of acid soluble collagen from calf skin were used: The thermal denaturation study was carried out with a product from Sigma Chemical Co. (Type III, lot No. 58C-8010) and the fibril formation was examined with one from Worthington Biochemical Corp. (Freehold, N. J., lot No. I1C781). The $0.5 \mathrm{M}$ acetic acid solution of these products was centrifuged at $10^{5} \times g$ for $1 \mathrm{hr}$, and then the supernatant was dialyzed against the same solvent containing $5 \% \mathrm{NaCl}$. The resultant precipitate was further purified by repeating the same procedures. Finally, the precipitate was exhaustively dialyzed against $10^{-4} \mathrm{M} \mathrm{HCl}$ and lyophilized. All these treatments were done at $4^{\circ} \mathrm{C}$ to avoid denaturation of the protein. Heavy water used was a spectrograde product $(99.75 \%)$ obtained from E. Merck AG. All other chemicals were special reagent grade products from Wako Pure Chemicals.

High pressure apparatus. The high-pressure apparatus utilizable up to 6,000 atm was purchased from Kobe Steel Ltd, (Kobe, Japan). This apparatus is equipped with high pressure windows of UV grade sapphire and terminals for electric conductivity measurements. The hydrostatic pressure was generated through silicone oil with a commercial hand pump. The pressure was measured with a bridge-type pressure gauge (Toyo Baldwin Co., model SS-8D-E). The temperature of the sample, controlled by circulating water of a given temperature through the jacket surrounding the exterior of the pressure vessel, was measured with a digital thermometer (Takara Kogyo, D221) with a sensor imbedded in the jacket. Temperature fluctuation in the equilibrated vessel was within $0.1^{\circ} \mathrm{C}$.

Thermal denaturation. The thermal denaturation of collagen was followed by "circular dichroism (CD) spectroscopy with a Jasco J-40A spectropolarimeter. For thermal denaturation under atmospheric pressure, a $0.01 \%$ collagen solution of a given $\mathrm{pH}$ adjusted with $0.01 \mathrm{M} \mathrm{HCl}$ was introduced into a water-jacketed cylindrical cell of $10 \mathrm{~mm}$ light path. The temperature of the sample was controlled by circulating water of a given temperature through the cell housing from a Sharp thermoelectric bath (model TE-12K). The temperature was measured with a digital thermometer, which was connected to a sensor immersed in the top part of the sample solution. The ellipticity at each new temperature was read $15 \mathrm{~min}$ after setting and was taken as a quasi-equilibrium value. In order to protect the protein from ultraviolet irradiation, the slit in the spectropolarimeter was closed in the times of temperature changing, and opened for only $60 \sim 90 \mathrm{sec}$ to read the ellipticity. On quenching the heat denatured sample at $20^{\circ} \mathrm{C}$, the ellipticity instantly recovered by $10 \%$ at most, followed by a negligibly slow recovery with time. The conversion ratio from helix to coil at temperature $T$ was calculated from the equation $\left(\theta^{20^{\circ} \mathrm{C}}-\theta^{T}\right) /$ $\left(\theta^{20^{\circ} \mathrm{C}}-\theta^{55^{\circ} \mathrm{C}}\right)$ by using the ellipticity, $\theta$, at $221 \mathrm{~nm}$. The temperature at the midpoint of the helix-coil transition was defined as the transition temperature, $T_{\mathrm{m}}$. The CD spectra are presented in terms of molar ellipticity, $[\theta]$, in $\mathrm{deg} \cdot \mathrm{cm}^{2} / \mathrm{dmol}$ with a mean residue molecular weight of 91.2.

Direct CD measurement under high pressure seems at present technically difficult due to the distortion effect caused by the pressure on the sapphire windows of the pressure vessel. Since the thermal denaturation of this collagen sample may be regarded approximately to be an irreversible process as mentioned above, the samples heatdenatured under high pressure were assayed at $25^{\circ} \mathrm{C}$ under atmospheric pressure as follows. Sample solutions were sealed in silicone tubes with glass stoppers. The tubes were placed in the pressure vessel at around $20^{\circ} \mathrm{C}$. After the pressure of the vessel was elevated to $4,300 \mathrm{~atm}$, the temperature was quickly raised to a fixed denaturation level (in most cases, $44.5^{\circ} \mathrm{C}$ and $49.0^{\circ} \mathrm{C}$ ). In spite of the large bulk of the pressure vessel, temperature equilibration required less than $25 \mathrm{~min}$. After the temperature equilibration, the pressure was rapidly dropped to a working one and the protein was denatured for 15 min under each condition. To stop the reaction, the system was again compressed to $4,300 \mathrm{~atm}$ while being rapidly cooling to about $20^{\circ} \mathrm{C}$ by use of a large amount of ice, followed by release of the pressure to $1 \mathrm{~atm}$. Immediately the samples were assayed by measuring the ellipticity at $221 \mathrm{~nm}$ with a $1 \mathrm{~mm}$ path-length cell. No significant time-dependent effects were observed in the ellipticity after decompression. The native fraction of collagen was estimated by comparing the ellipticity at each pressure with the ellipticities of completely native and denatured collagens. The pressure 
at $50 \%$ denaturation was determined from the pressure transition curve at each denaturation temperature.

Fibril formation. Purified collagen was dissolved in $5 \mathrm{~mm}$ acetic acid at twice the desired final concentration. All the experiments were done in phosphate buffer $(\mathrm{NaOH}-$ $\mathrm{KH}_{2} \mathrm{PO}_{4}, \mathrm{pH} 7.0$ ), maintaining the total phosphate concentration at $0.05 \mathrm{M}$. The ionic strength was adjusted by varying the $\mathrm{NaCl}$ content of the buffer. At zero time, fibril formation was initiated by completely mixing $1.5 \mathrm{ml}$ of collagen solution with $1.5 \mathrm{ml}$ of phosphate buffer incubated at a desired temperature. Keeping the temperature constant, a portion of the mixture was quickly introduced into a spectrophotometer cell $(5 \mathrm{~mm}$ lightpath) with a slide-type stopper. The cell was placed in the pressure vessel equilibrated at the working temperature, followed by quick compression of the vessel to the desired pressure. All these procedures were completed within approximately $2.5 \mathrm{~min}$. The process of fibril formation under pressure was monitored by recording the optical density at $400 \mathrm{~nm}$ as a function of time. The spectrophotometer used was a Hitachi model 139 whose sample chamber was replaced by the pressure vessel. As the progress curves depended on the age of collagen solutions, a series of experiments was done within 2 days using sample solutions of less than 10 days old.

\section{RESULTS}

\section{Pressure effects on thermal denaturation}

Figure 1 shows typical CD spectra of col-

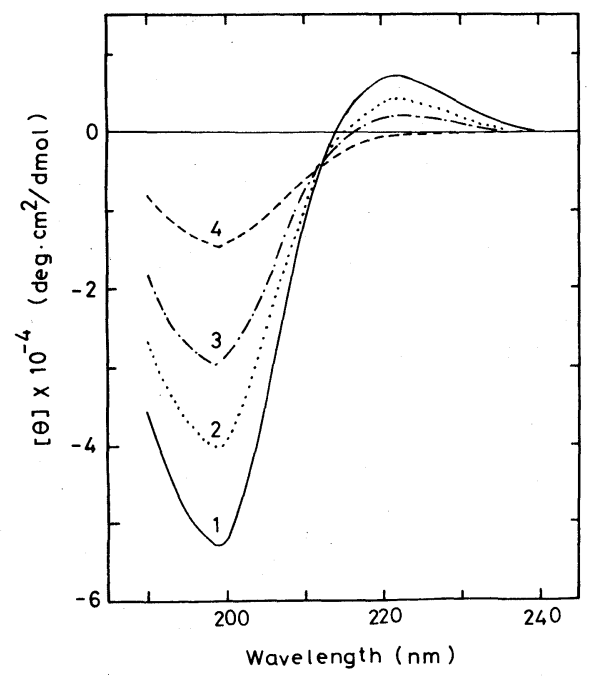

FIG. 1. Typical CD Spectra of Collagen Heat-denatured at $50^{\circ} \mathrm{C}$ under Various Pressures.

Curve 1, not heated (native form); curve 2, 3,100 atm; curve 3, 2,500 atm; curve 4, 1 atm. Protein concentration, $0.01 \%$. pH 4.0 . lagen denatured under various conditions. Native collagen has a positive peak at $221 \sim 222 \mathrm{~nm} \quad\left([\theta]=\sim 7,000 \mathrm{deg} \cdot \mathrm{cm}^{2} / \mathrm{dmol}\right)$ and a negative peak at $198 \sim 199 \mathrm{~nm}([\theta]=$ $\sim-53,000 \mathrm{deg} \cdot \mathrm{cm}^{2} / \mathrm{dmol}$ ), characteristic of the helix structure. The intensity of both peaks was significantly diminished on heating the sample at $50^{\circ} \mathrm{C}$ under $1 \mathrm{~atm}$. The samples heated under high pressure, however, did not show such a great depression of the peaks, indicating that the helix structure of collagen could be stabilized by pressure. The ellipticity at $221 \mathrm{~nm}$ was used as the measure of the helix content since the peak position was not affected by the denaturing conditions used.

Figure 2 shows the plots for the native fraction as a function of pressure at some

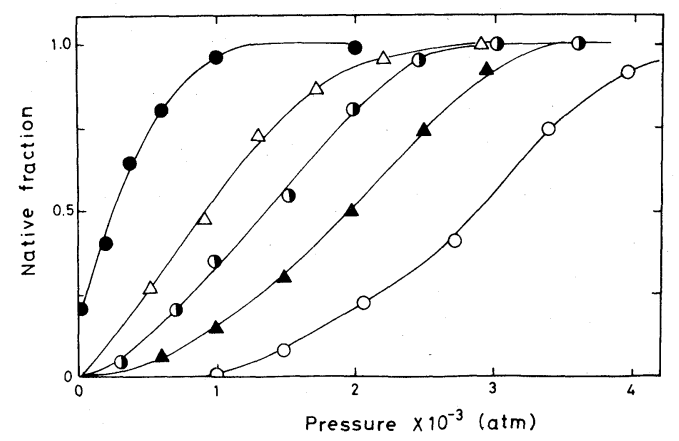

FIG. 2. Typical Pressure Transition Curves of Collagen.

(O) $\mathrm{pH} 4.0,40^{\circ} \mathrm{C}$; (O) $\mathrm{pH} 4.0,44.5^{\circ} \mathrm{C}$; (○) $\mathrm{pH} 4.0,49^{\circ} \mathrm{C}$; (A) $\mathrm{pH} 2.0,40^{\circ} \mathrm{C} ;(\triangle) \mathrm{D}_{2} \mathrm{O}, 44.5^{\circ} \mathrm{C}$.

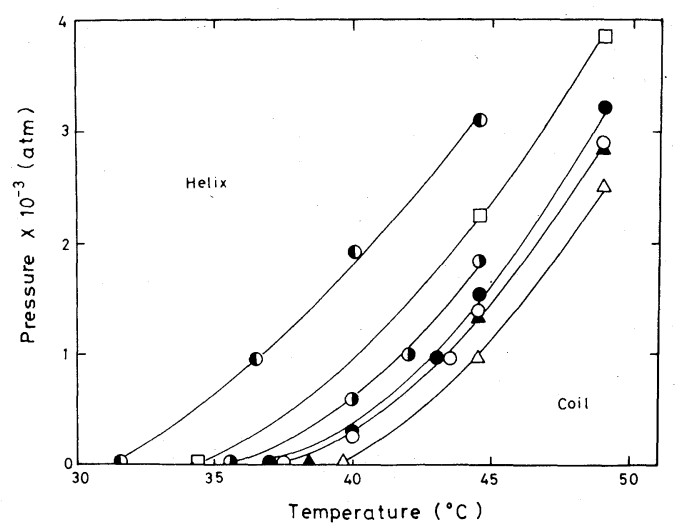

Fig. 3. Effects of Temperature and Pressure on the Structural Stability of Collagen.

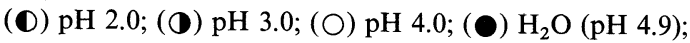
(口) $0.1 \mathrm{M} \mathrm{NaCl} ;(\Delta) 50 \% \mathrm{D}_{2} \mathrm{O} ;(\triangle) \mathrm{D}_{2} \mathrm{O}$. 
denaturation temperatures (pressure transition curves). High pressure restrains the thermal denaturation of collagen; e.g., this protein (pH 4.0) is completely denatured at $45^{\circ} \mathrm{C}$ under $1 \mathrm{~atm}$, but it remains native under high pressure above 3,000 atm. In Fig. 3 the values of pressure at $50 \%$ transition are plotted as a function of denaturation temperature. Although these temperature-pressure $(T-P)$ curves refer not to real equilibrium states but to apparent ones due to experimental limitations, they seem to present new important information on the stability of the collagen helix structure. The $T$ - $P$ diagrams appear to curve slightly upward with an almost identical positive slope independent of $\mathrm{pH}$ and additives used, suggesting that the effects of these solvent factors on the collagen structure are not largely modified by pressure. The slope, $(\mathrm{d} T / \mathrm{d} P)$, assumed a constant value of $2.9 \times$ $10^{-3} \mathrm{deg} / \mathrm{atm}$ in the pressure region of 1,500 to $2,500 \mathrm{~atm}$, which seemed to increase to $(4.6 \sim 5.5) \times 10^{-3} \mathrm{deg} / \mathrm{atm}$ in lower pressure regions below $1,000 \mathrm{~atm}$.

\section{Pressure effects on fibril formation}

Figure 4 shows the turbidity changes as a function of time for self-assembling of collagen under several different pressures. All turbidity curve show a characteristic lag period followed by a sigmoidal increase in turbidity, indicating that the fibril formation under high pressure proceeds in two consecutive phases, a nucleation phase and a growth phase, just as under atmospheric pressure. ${ }^{19)}$ The final opacity, which did not seem to vary greatly under pressure in most cases, could not be determined, since it was difficult to keep each sample under high pressure for a few days without sample aging as mentioned in experimental procedures. Therefore, the maximum slope in the turbidity curves, $\mathrm{d} E / \mathrm{d} t$, usually corresponding to the slope of their linear portion, was taken as a relative measure of the growth rate. The rate of nucleation was approximated as the reciprocal value of lagphase time, $t_{n}$, which was obtained by extrapolating the line of the maximum slope to the

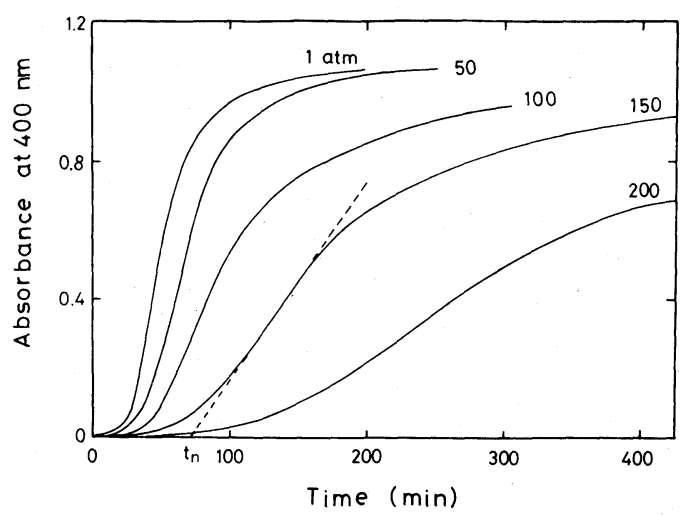

FIG. 4. Turbidity Change as a Function of Time for Collagen Fibril Formation at $25^{\circ} \mathrm{C}$ under Various Pressures.

The numbers with the curves represent the pressure applied. Collagen concentration, $0.08 \% ; \mathrm{pH} 7.0$; ionic strength, 0.12 . The maximum slope of the turbidity curve (broken line) was used for comparison of the growth rates. The nucleation time, $t_{n}$, was obtained by extrapolating the line of the maximum slope to the abscissa.

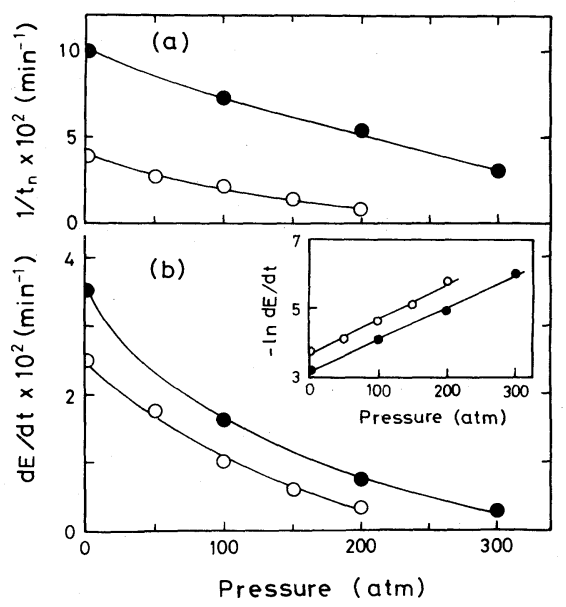

FIG. 5. Pressure Effects on Fibril Formation Kinetics, Nucleation Rate (a) and Growth Rate (b), at $25^{\circ} \mathrm{C}$.

Protein concentration, $0.08 \%$; $\mathrm{pH} 7.0$; ionic strength, 0.10 (O) and $0.12(\bigcirc)$. The inset in the figure shows logarithmic plots of growth rate against pressure. From the slope of the linear lines, the apparent activation volume was estimated to be $230 \mathrm{ml} / \mathrm{mol}$ and $260 \mathrm{ml} / \mathrm{mol}$ at ionic strengths of 0.10 and 0.12 , respectively.

abscissa in time scale (see Fig. 4). The thus obtained $t_{n}$ involves an inaccuracy of $2.5 \mathrm{~min}$, the time required for pressure setting.

The values of $\mathrm{d} E / \mathrm{d} t$ and $1 / t_{n}$ are plotted as a function of supplied pressure in Fig. 5. 


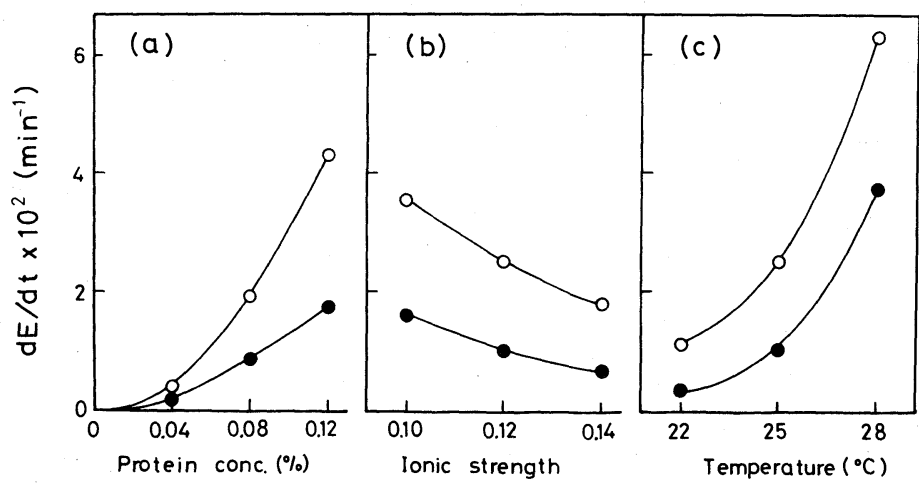

FIG. 6. Effects of Protein Concentration, Ionic Strength and Temperature on Fibril Formation Kinetics under $1 \mathrm{~atm}(\bigcirc)$ and $100 \mathrm{~atm}(\bigcirc)$.

(a) Effect of protein concentration at $25^{\circ} \mathrm{C}$ and an ionic strength of 0.12 . (b) Effect of ionic strength at $25^{\circ} \mathrm{C}$ and a protein concentration of $0.08 \%$. (c) Effect of temperature at a protein concentration of $0.08 \%$ and an ionic strength of 0.12 .

Evidently, the fibril formation is retarded by pressure in both the nucleation and growth phases, the pressure effect being more significant in lower pressure regions. At higher pressure of 1,000 and 2,500 atms, no appreciable turbidity increase was observed during our routine measurement period (about $6 \mathrm{hr}$ ). As shown in the inset of Fig. 5, there is a linear relationship between $\ln (\mathrm{d} E / \mathrm{d} t)$ and pressure, suggesting that the activation volume of fibril formation process, $\Delta V^{\neq}$, is approximately constant over the pressure region examined. The apparent values of $\Delta V^{\neq}$calculated from the slopes were $230 \mathrm{ml} / \mathrm{mol}$ and $260 \mathrm{ml} / \mathrm{mol}$ at ionic strengths of 0.10 and 0.12 , respectively.

The values of $\mathrm{d} E / \mathrm{d} t$ for 1 and 100 atms are plotted as function of protein concentration, ionic strength and temperature in Fig. 6, where the effect of protein concentration was investigated with separately-prepared collagen solutions. The corresponding nucleation rates were shown to depend in a similar way on these solution factors.

\section{DISCUSSION}

The present study revealed that the thermal denaturation and fibril formation of collagen are depressed by pressure. The pressure stabilization of the collagen triple helix is very interesting since most globular proteins are denatured by pressure in these high pressure regions. ${ }^{20 \sim 22)}$ The $T$-P planes for the stability of collagen are rather similar to those for deoxyribonucleic acid (DNA) whose double helix is also stabilized by pressure. ${ }^{23,24)}$ On the other hand, prevention of the fibril formation is accomplished in relatively low pressure regions up to $500 \mathrm{~atm}$ in a similar way as for selfassembling of tubulin, actin and myosin. ${ }^{25 ~ 28)}$ Detailed thermodynamic discussion of these results is outside the scope of this study because of the experimental limitation. Nevertheless, it seems appropriate at the present stage to discuss the volume changes involved in both types of transitions, the helix-tocoil transition and the fibril formation.

The positive slopes observed in $T-P$ curves (Fig. 3) indicate that the volume change of the helix-to-coil transition of collagen is positive in water. If we assume the values of $2.9 \times 10^{-3}$ $\mathrm{deg} / \mathrm{atm}$ for $(\mathrm{d} T / \mathrm{d} P)$ and $0.036 \mathrm{cal} / \mathrm{deg} \cdot \mathrm{g}$ for the entropy change, $\Delta S,{ }^{29)}$ then the volume change under consideration, $\Delta V$, would be about $4.3 \times 10^{-3} \mathrm{ml} / \mathrm{g}$ with the ClausiusClapeyron equation, $(\mathrm{d} T / \mathrm{d} P)=\Delta V / \Delta S$. To our knowledge, there have been only two lots of limited data on the volume change of this process, probably because the collagen solution is too viscous to be used for densimetric or dilatometric measurements. Flory and Garrett ${ }^{16)}$ found an increase in latent volume 
(ca. $5 \times 10^{-3} \mathrm{ml} / \mathrm{g}$ ) for the thermal shrinkage of tendon collagen fibers swollen in ethylene glycol. Although this value seems to agree with our predicted one, this coincidence may be rather fortuitous because their volume change refers to the macroscopic interprotofibrillar space within the crystalline phase and it does not involve the contribution of hydration. On the other hand, Fessler and Hodge ${ }^{17)}$ showed by density gradient ultracentrifugal analysis that gelatin is denser than collagen fibrils and collagen fibrils are denser than the collagen monomer. This finding suggests a negative volume change for the transition of collagen monomer to gelatin, the thermal denaturation. When referring to this result, however, it should be noted that the densities they measured were apparent ones involving the pressure effects on the partial specific volume of the protein itself, ${ }^{30)}$ preferential solvation, and on fibril formation as demonstrated in this study.

Cassel and Christensen ${ }^{18)}$ found by dilatometric techniques that the volume change due to fibril formation is $8 \times 10^{-4} \mathrm{ml} / \mathrm{g}$, positive and independent of solvent conditions. The pressure-induced retardation of fibril formation mentioned above supports the positive volume change in this association process. Such inhibition of fibril formation is not caused by any denaturation of the collagen molecule since the triple helix is stabilized, instead of destabilized, by pressure. As formation of a higher-ordered molecular state is usually accompanied by a volume decrease, such an increase in volume would be mainly due to a reduction in number of hydration water involved in the fibril formation process. It is interesting to note that the estimated apparent activation volume of fibril formation, $230 \sim 260 \mathrm{ml} / \mathrm{mol}$, is close to the total volume change, $240 \mathrm{ml} / \mathrm{mol}$, which was calculated by multiplying the molecular weight of collagen $\left(3 \times 10^{5}\right)$ by the volume change obtained by Cassel and Christensen. This coincidence leads to the idea that the molecular structure of the activated state in the fibril formation process is close to that of fibrils. This idea is consistent with the conclusion of Wood and Keech that most of the fibrils are formed during the lag period. ${ }^{31)}$

Why can high pressure stabilize the triple helix and destabilize the fibrils of collagen? One can give only speculative explanations, at present, for this problem because of the complicated response of pressure to stabilizing forces of the protein structure. An important finding in the present work is that the volume change due to the helix-to-coil transition of collagen is positive in spite of the increased hydration. This would be expected if the volume change is positive for the process of breaking peptide-peptide hydrogen bonds and replacing them by peptide-water hydrogen bonds, as suggested by the observation of Suzuki et al., that the solubility of diketopiperazine in water decreases with pressure. ${ }^{32)}$ It is known in this regard that the hydrophobic interaction between detergent or $n$-alkane molecules is weakened in the presence of high pressure. ${ }^{33,34)}$ The pressure retardation of the fibril formation may be explained in terms of a lessened hydrophobic interaction between collagen monomers, probably caused by nonpolar pyrrolidine residues present in a large proportion on the protein surface. Although these explanations for the pressure effects should be checked by further detailed studies in this area, the preliminary observations in this study seem to support the hypothesis that the protein-solvent interaction plays an important role in the stabilization of the helix and fibril structure of collagen.

The pressure stabilization of the monomer structure of collagen against heat and selfassembly, whatever the mechanism may be, is a matter of interest from biological or physiological aspects. It is well known that the thermal denaturation or the shrinkage temperature of collagen from a wide range of species increases as the temperature of their environment rises. ${ }^{35,36)}$ This phenomenon has been explained in terms of the different content and distribution of proline and hydroxyproline residues along single polypeptide chains. The present study is positive substantiation of the 
possibility that the environmental pressure of deep-sea fishes may also affect the structural stability of their collagens as a consequence of the biosynthesis of procollagen molecules and/or the pressure-controlled in vivo fibril formation of collagen.

Acknowledgment. The authors wish to thank Mr. Norihisa Umikawa for his experimental assistance.

\section{REFERENCES}

1) A. R. Haly and J. W. Snaith, Biopolymers, 10, 1681 (1971).

2) C. A. J. Hoeve and R. R. Kakivaya, J. Phys. Chem., 80, 745 (1976).

3) M. H. Pineri, M. Escoubes and G. Roche, Biopolymers, 17, 2799 (1978).

4) A. Yonath and W. Tranb, J. Mol. Biol., 43, 461 (1969).

5) R. D. B. Fraser, T. P. Macrae and E. Suzuki, "Fibrous Proteins: Scientific, Industrial and Medical Aspects," Vol. 1, ed. by A. D. Parry and L. K. Creamer, Academic Press, New York, 1979, pp. $179 \sim 206$.

6) J. Schnell, Arch. Biochem. Biophys., 127, 496 (1968).

7) B. S. Harrap, Int. J. Protein Res., 1, 245 (1969).

8) A. E. Russell and D. R. Cooper, Biochemistry, 8, 3980 (1969).

9) A. E. Russell, Biochem. J., 131, 335 (1973).

10) D. Eagland, "Water," Vol. 4, ed. by F. Franks, Plenum Press, New York, 1975, Chapter 5.

11) P. L. Privalov and E. I. Tiktopulo, Biopolymers, 9, 127 (1970).

12) A. Cooper, Biochem. J., 118, 355 (1970).

13) J. M. Rifkind and J. Applequist, J. Am. Chem. Soc., 90, 3650 (1968).

14) F. R. Brown III, A. J. Hopfinger and E. R. Blout, $J$. Mol. Biol., 63, 101 (1972).
15) P. L. Privalov and G. M. Mrevlishvili, Biofizika, 12, 23 (1967).

16) P. J. Flory and R. R. Garrett, J. Am. Chem. Soc., 80, 4836 (1958).

17) J. H. Fessler and A. J. Hodge, J. Mol. Biol., 5, 446 (1962)

18) J. M. Cassel and R. G. Christensen, Biopolymers, 5, 431 (1967).

19) G. C. Wood, Biochem. J., 75, 598 (1960).

20) J. F. Brants, R. J. Oliveira and C. Westort, Biochemistry, 9, 1038 (1970).

21) S. A. Hawley, Biochemistry, 10, 2436 (1971).

22) A. Zipp and W. Kauzmann, Biochemistry, 12, 4217 (1973).

23) K. Suzuki, Y. Miyosawa and Y. Taniguchi, J. Biochem., 69, 595 (1971); J. Biochem., 72, 1087 (1972).

24) S. A. Hawley and R. M. Macleod, Biopolymers, 13, 1417 (1974)

25) E. D. Salmon, Science, 189, 884 (1975).

26) Y. Engelborghs, K. A. H. Heremans, L. C. M. De Maeyer and J. Hoebeke, Nature, 259, 686 (1976).

27) T. Ikkai and T. Ooi, Biochemistry, 8, 2615 (1969).

28) J. S. Davis, Biochem. J., 197, 301 (1981).

29) P. E. McClain and E. R. Wiley, J. Biol. Chem., 247, 692 (1972).

30) K. Gekko and H. Noguchi, J. Phys. Chem., 83, 2706 (1979).

31) G. C. Wood and M. K. Keech, Biochem. J., 75, 588 (1960).

32) K. Suzuki, M. Tsuchiya and H. Kadono, Bull. Chem. Soc. Jpn., 43, 3083 (1970).

33) T. S. Brun, H. Høiland and E. Vikingstad, J. Colloid Interface Sci., 63, 89 (1978).

34) N. Nishikido and M. Tanaka, Surface (Hyomen), 17, 215 (1979).

35) B. J. Rigby, Nature, 219, 166 (1968).

36) B. J. Rigby and C. L. Prosser, Comp. Biochem. Physiol., 52B, 89 (1975). 